\title{
Molecular Profiling of Core Immune-Escape Genes Highlights LCK Has Potential to Reshape TME in Melanoma
}

\section{Duoli Zhang}

Southwest Medical University

\section{Zhuo Zhang}

Southwest Medical University

\section{Shixin Xiang}

Southwest Medical University

\section{Mintao Xiao}

Southwest Medical University

\section{Yao Zhang}

Southwest Medical University

\section{Suwit Duangmano}

Chiang Mai University

\section{Xu Wu}

Southwest Medical University

\section{Mingxing Li}

Southwest Medical University

\section{Fukuan Du}

Southwest Medical University

\section{Yu Chen}

Southwest Medical University

\section{Xiao Yang}

Southwest Medical University

\section{Yueshui Zhao}

Southwest Medical University

\section{Yuan Zheng}

Neijiang Health and Health Vocational College

\section{Qinglian Wen}

Affiliated Hospital of Southwest Medical University

Jing Li

Hospital (T.C.M) Affiliated to Southwest Medical University

Jing Shen 
Southwest Medical University

Zhangang Xiao ( $\sim$ zhangangxiao@swmu.edu.cn )

Southwest Medical University

\section{Zhongming Yang}

Hospital (T.C.M) Affiliated to Southwest Medical University

\section{Research Article}

Keywords: ssGSEA, CIBERSORT, Prognostic model, immune infiltration, melanoma

Posted Date: September 28th, 2021

DOI: https://doi.org/10.21203/rs.3.rs-923242/v1

License: (c) (i) This work is licensed under a Creative Commons Attribution 4.0 International License. Read Full License 


\section{Abstract}

Background: The game between the immune system of the organism and the tumor is a dynamic course of events. Once the escape phase is reached, the tumor will overcome the limitations of the immune system and the tumor will progress.

Objective: This study aimed to determine the potential tumor environment-based prognostic biomarkers related to immunotherapy in melanoma.

Methods: 471 tumor samples and 398 normal samples were extracted from The Cancer Genome Atlas and The Genotype-Tissue Expression. Furthermore, a core set of immune-escape genes were collected from previous studies and a set of immune-related genes were obtained from IMMPORT database. Through overlapping these two sets of genes, a set of core immune-escape related genes were identified. In the next place, we conducted a systematic analysis of core immune-escape related genes through The Cancer Genome Atlas cohort and identified independent prognostic factors in melanoma. Through CIBERSORT, ssGSEA algorithm and TIMER database, we explored the potential of independent prognostic factors to reshape the tumor microenvironment.

Results: Through Kaplan-Meier as well as univariate cox regression analysis of expression profiles and clinical information obtained from the TCGA cohort, we found that high LCK expression was associated with prolonged overall survival. In addition, the expression level of LCK was significantly correlated with the dysregulation of the infiltration level of immune cells in the tumor microenvironment.

Conclusions: In this study, we determined LCK as a biomarker that is significantly associated with tumor environment and has significant prognostic significance. Meanwhile, immunotherapeutic approaches targeting LCK in tumor cells may provide a new perspective for the treatment of melanoma.

\section{Background}

Melanoma is the type of skin cancer with the highest levels of mortality, and it is more dangerous because it can spread to other parts of the body if not caught and treated early[1]. The global incidence of melanoma has steadily increased over the years; in the United States approximately 100,000 new cases of invasive melanoma will be diagnosed in 2021 and about 7,000 of those melanoma patients will die from the disease[2, 3]. The diagnosis of melanoma is a challenge for both dermatologists and oncologists[4]. Treatment options for melanoma include traditional surgery, chemotherapy, radiotherapy and administration of small molecule inhibitors, especially immunotherapy targeting immune checkpoints, which has been shown to be highly effective. Currently, dual immune checkpoint inhibitor (ICI) with nivolumab and ipilimumab offers the best first-line treatment option for patients with BRAF-wt and -mutated for advanced melanoma[5]. In addition, the triple combination of anti-PD-1/PD-L1, antiBRAF and anti-MEK therapies are also attracting attention. 
The immune system has a crucial role in the development and treatment of cancer. Adaptive immunity can prevent or constrain cancer through immunosurveillance, whereas innate immunity and inflammation tend to promote tumorigenesis and malignant development of nascent cancer[6]. Cancer immunoediting is the process by which the immune system both constrains and promotes tumor development, and it proceeds through three phases: elimination, equilibrium and escape[7]. Cancer is a highly heterogeneous disease involving complex interactions between the malignant cells and the tumor microenvironment (TME)[8]. Cancer cells have evolved multiple mechanisms to evade immune recognition or to modulate immune cell function[9]. TME, a mixture that consists of mesenchymal cells, tumor-infiltrating immune cells (TIICs), endothelial cells, extracellular matrix molecules and inflammatory mediators[10]. Increasing evidence demonstrated the importance of the TME in the tumor development[11]. Collaborative interactions between cancer cells and their supporting cells contributed to the malignant phenotypes of cancer, such as immortal proliferation, resisting apoptosis, and evading immune surveillance. Therefore, the TME significantly influences therapeutic response and clinical outcome in cancer patients[12, 13]. TME is closely correlated to tumor initiation, progression and prognosis[14]. The composition of the tumor-infiltrating population reflects the mechanisms underlying anticancer immune responses and can help identify novel prognostic signatures[8].

Cell type Identification By Estimating Relative Subsets Of RNA Transcripts (CIBERSOT) is an algorithm developed by Aaron M. Newman et al. in 2015, based on the principle of linear support vector regression (linear support vector regression) to deconvolve the expression matrix of human immune cell subtypes in an algorithm designed to determine the proportion of 22 immune cell compositions in each sample. thereby assessing the immune infiltration of tumor microenvironment species[15]. Furthermore, single cell gene set enrichment analysis (sSGSEA) algorithm is a new algorithm developed on the basis of GSEA, first proposed in 2009 by David A. Barbie et al. This was achieved through a single-sample extension of GSEA, designed to allow researchers to define an enrichment score that would allow one to obtain the level of infiltration of 29 immune cell species in each sample[16]. In this study, we obtained a set of genes related to immune escape from an article and identified differentially expressed proteins regulated by immune-escape related genes through the cbiportal website, identified protein-coding genes highly associated with prognosis by constructing a prognostic model, and finally described the role played by independent prognostic factors in remodeling TME using CIBERSORT and ssGSEA.

\section{Materials And Methods}

\section{Data collection}

Gene expression profiles, clinical phenotype information and somatic mutation data of melanoma were extracted from The Cancer Genome Atlas (TCGA, https://portal.gdc.cancer.gov) database. Melanoma sample information was obtained from TCGA and Genotype-Tissue Expression Project (GTEx). The expression profile data are presented in TPM form and are normalized by $\log 2(x+0.001)$. TCGA data were downloaded through the "TCGAbiolinks" package through R project and the above pre-processing of gene expression profiles was carried out in $\mathrm{R}$ project. 


\section{Identification and characterization of immune-related genes in melanoma}

A core set of immune-escape related gene were extracted from papers[17]and interference with these genes alone will increase the sensitivity or resistance of cancer cells to Cytotoxic T lymphocyte (CTL)mediated toxicity. Furthermore, a set of immune-related genes was acquired from the IMMPORT (https://www.immport.org/) database which were curated through functions and gene ontology terms. To be next, overlapping these two sets of gene to obtain core immune-escape related genes. In addition, a combination of univariate cox regression analysis and Kaplan-Meier survival analysis was utilized to evaluate the prognostic value of the intersecting genes, while differential expression analysis of crossover genes in normal and tumor tissues was conducted in order to determine primary screening genes. Subsequently, we visualized the somatic mutation data in Mutation Annotation Format (MAF) using the "maftools" R package, which provides a large amount of commonly used analysis and visualization modules in cancer genomic studies[18].

\section{Identification of differentially expressed proteins and their enrichment pathways, analysis of protein- protein interactions.}

The cBioPortal for Cancer Genomics(http://cbioportal.org) provides a Web resource for exploring, visualizing, and analyzing multidimensional cancer genomics data[19]. The protein expression changes (both unphosphorylated and phosphorylated proteins) in melanoma were analyzed in cBioPortal through Reverse Phase Protein Array (RPPA)[20]. Proteins with significant differences (-log10 P-value > 1.3) are indicated by dots of different colors (The under-expression group is indicated in green and the overexpression group is indicated in red). The R package "ggplot2" and "ClusterProfiler" were then used to perform enrichment analysis of these protein-coding genes and visualize the results, which contains Gene Ontology (GO) enrichment analysis of Cellular component (CC), Biological process (BP) and Molecular function (MF) as well as Kyoto Encyclopedia of Genes and Genomes (KEGG) enrichment analysis. STRING database (https://string-db.org/cgi/input.pl) can collect and integrate known and predicted protein-protein association data[21]. In this study, we used STRING database to construct Protein-Protein Interaction (PPI) network with minimum required interaction score 0.4 and disconnected nodes in the network were hide. Also, we exhibited the nodes with the top 60 highest connectivity in the network. At last, network was visualized through Cytoscape software and three important subnetworks were selected by MCODE which is a plugin within Cytoscape with a degree cut-off $=2$, node score cut-off $=0.2, k$-core $=$ 2 , and max Depth $=100$ as the criterion.

\section{Risk modeling and screening of independent prognostic factors}

The melanoma patients from TCGA were divided into a training set (TRS) and a testing set (TES). The TRS was used to construct a prognostic risk model of melanoma and the TES was utilized to valid the predictive capability of this model. The R package "survival" and "UpSetR" was used to perform univariate cox regression analysis to screen for protein-coding genes for significant association with overall survival in the TCGA-SKCM cohort. Protein-coding genes with $p$-value less than 0.05 were used for further analysis, and the fit was reduced by least absolute shrinkage and selection operator (LASSO) 
regression analysis to obtain fewer representative eigenvalues. 1000-round cross-validation for tuning parameter selection was used to prevent overfitting and the partial likelihood deviance met the minimum criteria[22]. The screened protein-coding genes were then subjected to multivariate cox proportionalhazard analysis through R package "survival" in R project to identify independent prognostic factors ( $\mathrm{p}$ value less than 0.05 ). In addition, three hub-independent prognostic factors were determined by overlaying the nodes with the top 60 connectivity in PPI with independent prognostic factors.

\section{Validation and analysis of risk prognostic models}

Risk score were calculated for each patient sample through the coef values from the multivariate cox proportional-hazards analysis results and the expression levels of independent prognostic factors in the results of multivariate analysis with the following formula:

[Risk Score (each patient $)=\sum_{i}$ Expression $\left.\left(\mathrm{mRNA}_{\mathrm{i}}\right) \times \operatorname{Coefficient}\left(\mathrm{mRNA}_{\mathrm{i}}\right)\right]$ Patients were then divided into high- and low-risk groups according to the median risk score, and a risk score model was developed. Then, ROC curves are created through the R package "pROC" to verify the accuracy of the risk model, with larger Area Under Curve (AUC) representing more accurate models. This risk model is then subjected to a series of analyses based on the established risk model. First of all, risk factor analysis was conducted through the online mapping site HIPLOT (https://hiplot/com.cn) to evaluate the association between patient survival status and expression level of independent prognostic factors and risk scores. Furthermore, we divided the sample into high- and low-risk score groups and assessed the ability of the risk scores to predict patient prognosis by Kaplan-Meier survival analysis. At last, through TIMER, a web server for comprehensive analysis of tumor-infiltrating immune cells, data on the infiltration level of six kinds of immune cells were downloaded[23]. Differential infiltration level of six kinds of immune cell in high- and low-risk score subgroups was analyzed.

\section{The expression, survival and immune microenvironment analysis of Hub-independent prognostic factors}

Prognostic value of all hub-independent prognostic factors and differential expression in tumor and normal tissues were analyzed in the TCGA cohort. Moreover, to further demonstrate that the accuracy of the above results, Gene Expression Profiling Interactive Analysis (GEPIA, http://gepia.cancer-pku.cn/), a web server for cancer and normal gene expression profiling and interactive analysis[24], was utilized to validated above results. Furthermore, the association between 6 kinds of immune cells and hubindependent prognostic factors was analyzed through TIMER database. Similarly, we quantified the level of infiltration of 29 kinds of immune cell types through the ssGSEA algorithm in the R package "GSVA" and then assessed the relationship between the infiltration level of 29 kinds of immune cell and the mRNA expression of three hub-independent prognostic factors.

\section{Correlation of three hub-independent prognostic factors with the common ICPs}


To evaluate the immunotherapy responses through three hub-prognostic factors expression, we explore the correlation of three hub-independent prognostic factors expression level with common ICPs[25], which contains recombinant cluster of differentiation 276 (CD276), interleukin 6 (IL6), transforming growth factor beta-1 (TGFB1), T cell immunoreceptor with Ig and ITIM domains (TIGIT), programmed cell death 1 (PDCD1), lymphocyte activation gene-3 (LAG3), programmed cell death 1 ligand 1 (PD-L1), hepatitis a virus cellular receptor 2 (HAVCR2), cytotoxic T lymphocyte antigen 4 (CTLA4), v-set immunoregulatory receptor (c10orf54), c-c motif chemokine ligand 2 (CCL2), C-X-C motif chemokine receptor 4 (CXCR4). Furthermore, gene set enrichment analysis (GSEA) was conducted in LCK high- and low-expression cohorts.

\section{The relationship between LCK with the proportion of (tumor-infiltrating immune cells) TICs}

In this study, CIBERSORT algorithm was used to evaluate the proportion of 22 kinds of immune cells in each sample, which allowed us to assess the correlation between the mRNA expression of LCK and the level of infiltration of immune components. Only samples with p-value less than 0.05 were included in the next further analysis.

\section{Statistical analysis}

Analysis of variance between two groups was performed by Student's t-test, and analysis of variance between multiple groups $(n=>3)$ was performed by one-way ANOVA. Prognostic survival analysis was conducted through Kaplan-Meier as well as univariate and multivariate risk proportional regression models. p-values were calculated through log-rank test. p-values less than 0.05 were considered to be significantly different. Correlation coefficients were calculated using Spearman, and correlation coefficients with absolute values greater than 0.3 were considered to be correlated. The above statistical analyses were performed in R project as well as in PRISM 7.0 software.

\section{Results}

\section{Landscape of immune-related genes in melanoma}

The entirety of this study was accomplished through the following flowchart (Supplementary 1). A set of immune-escape related genes and a set of immune-related genes were extracted from the literature and from the IMMPORT website respectively, which contains 182 genes and 2483 genes respectively in total. Overlapping these two gene sets, 31 core immune-escape related genes (Table 1) were determined as subjects for further analysis (Fig. 1A). In addition, 472 (including 471 tumor samples and 1 normal sample) and 398 normal samples were obtained from the TCGA and GTEx databases, respectively. Results of Kaplan-Meier survival analysis of core immune-escape related genes as well as univariate cox analysis showed that except for CALR, TNFRSF1A, HDAC1, JAK1 and TFRC, which were not significantly different, and MAPK1, which was an unfavorable prognostic factor, the rest of all the core immuneescape genes were favorable prognostic factors, and all of them were significantly different (Fig. 1B, Supplementary 2). Furthermore, the results of differential expression analysis of core immune-escape 
related genes in tumor and normal tissues showed that IFNGR1, JAK2, SOCS1, IKBKG, JAK1, TNFAIP3, TNFRSF1A, FAS, IKBKG, TBK1 and TGFBR2 were highly expressed in normal tissues, while the expression of the remaining genes were upregulated in tumor tissues, all of the above differential expression analysis results were significantly different (p-value< 0.05) (Fig. 1C). Genes with significantly different results in both expression difference analysis and prognostic analysis were initially screened out for inclusion in the next analysis. Somatic mutation profiles of 467 melanoma patients downloaded from TCGA database were analyzed and visualized via the "maftools" R package[26]. The results of mutation profiling of the primary screening genes showed that the mutation rate of all primary screening genes was low (Fig. 1D).

Table 1

A list of core immune-escape related genes.

\section{Gene Symbol}

ADAR, B2M, BECN1, CALR, ERAP1, FAS, HDAC1, IFNAR1, IFNAR2, IRF1, IRF9, IFNGR1, IFNGR2, IKBKG, IKBKB, JAK1, JAK2, MAPK1, PDLA3, PSMB8, SOCS1, STAT1, TAP1, TAP2, TAPBP, TBK1, TFRC,

TGFBR2, TNFAIP3, TNFRSF1A, TNFRSF1B

Table 2. Representative Gene Ontology results of these three subnetworks.

\begin{tabular}{|c|c|c|c|}
\hline Category & Term & Count & p-value \\
\hline \multicolumn{4}{|l|}{ Subnetwork 1} \\
\hline GOTERM_BP_DIRECT & Regulation of autophagy & 15 & $7.66 \mathrm{E}-12$ \\
\hline GOTERM_CC_DIRECT & Protein kinase complex & 5 & $1.19 \mathrm{E}-05$ \\
\hline GOTERM_MF_DIRECT & Ptotein tyrosine kinase activity & 8 & $7.34 \mathrm{E}-09$ \\
\hline \multicolumn{4}{|l|}{ Subnetwork 2} \\
\hline GOTERM_BP_DIRECT & $T$ cell activation & 7 & $8.43 \mathrm{E}-07$ \\
\hline GOTERM_CC_DIRECT & Extrinsic component of membrane & 5 & $1.92 \mathrm{E}-05$ \\
\hline GOTERM_MF_DIRECT & Cytokine receptor binding & 3 & 0.00398 \\
\hline \multicolumn{4}{|l|}{ Subnetwork 3} \\
\hline GOTERM_BP_DIRECT & $\begin{array}{l}\text { B cell activation involved in } \\
\text { immune }\end{array}$ & 4 & $2.99 \mathrm{E}-06$ \\
\hline GOTERM_CC_DIRECT & CD40 receptor complex & 2 & $7.88 \mathrm{E}-05$ \\
\hline GOTERM_MF_DIRECT & Receptor tyrosine kinase binding & 3 & 0.00011 \\
\hline
\end{tabular}

The differentially expressed proteins under the regulation of primary screening genes are mainly enriched in multiple immune-related pathways and functional

Identification of differentially expressed proteins regulated by primary screening genes through the RPPA module of the cBioPortal website and 164 differentially expressed proteins were obtained in total. Subsequently, GO and KEGG enrichment analysis of these protein-coding genes was conducted through the "ClusterProfile" package in R project. The pathway enrichment results showed that these protein- 
coding genes were mainly enriched in EGFR tyrosine kinase inhibitor resistance, ErbB signaling pathway, PI3K-Akt signaling pathway and some immune-related pathways (including $T$ cell receptor signaling pathway, Natural killer cell mediated cytotoxicity and so on) (Fig. 2A). GO enrichment analysis showed that these protein-coding genes were mainly enriched in the regulation of apoptotic signaling pathway and regulation of mitochondrion organization in $\mathrm{BP}$, chromosome, telomeric region and mitochondrial outer membrane in CC, phosphatase binding and ubiquitin-like protein ligase binding in MF (Fig. 2B). Through STRING database, protein-protein interaction network was constructed, which contains 188 nodes and 3670 edges in summary. After that, we filtered out the top 60 nodes in the entire network in terms of connectivity (Fig. 2C). Afterwards, we used the MCODE plugin in Cytoscape software in order to filter out the three important sub-networks of the network (Fig. 2D). Finally, GO enrichment analysis was performed on the nodes in each of the three subnetworks respectively, and the results showed that the main enrichment was in terms related to immunization (Table 2).

\section{Construction and evaluation of the melanoma-related risk signature which are predictive of prognosis}

The samples are divided into a TRS and a TES according to an approximate ratio of 1:1 (The TRS has 228 samples and the TES has 226 samples). The result of univariate cox regression analysis of TRS showed that a total of 15 prognostic factors were determined, which means changes in the expression levels of these 15 protein-coding genes affect the prognosis of patients. In addition that, except CDKN1B, LCK, and RICTOR which were favorable prognostic factors, the rest of these prognostic factors were associated with reduced overall survival (the $p$-value of the above results were less than 0.05) (Fig. 3A). Subsequently, prognostic factors were subjected to LASSO regression analysis and 10 representative protein-coding genes were determined (Fig. 3B). Multivariate cox regression analysis was conducted within these 10 representative protein-coding genes obtained from LASSO regression analysis and six independent prognostic factors were finally identified which were related to prognosis in melanoma. In summary, all four independent prognostic factors were associated with reduced overall survival, except for LCK and RICTOR, which were favorable factors, although the results for RICTOR were not significantly different (Fig. 3C). At last, three hub-independent prognostic factors were obtained by overlapping the independent prognostic factors with significant differences in the multivariate results and the top 60 nodes with the highest connectivity in the protein interactions network (Fig. 3D).

\section{Risk-prognosis models constructed by independent prognostic factors may prolong overall survival by affecting immune cell infiltration in TME}

Through analyzing the independent prognostic factors in the TRS, a risk prognostic model was constructed in the TRS and TES, using which the prognosis of patients could be effectively predicted. In the TRS and TES, ROC curves were established to validate the accuracy of the risk model, with AUC values equal to 0.683 and 0.657 , respectively, indicating that the model has good accuracy (Fig. 4A). After that, the results of the risk factor analysis showed that the number of patient deaths clustered significantly as the risk score increased, indicating that the risk score can effectively predict the prognosis of patients. In both TRS and TES, LCK and RICTOR were higher expressed in the low-risk group, while 
FOXM1, KIT, EGFR, SMAD1 were higher expressed in the high-risk group (Fig. 4B). Survival analysis in the high- and low-risk score groups showed that the high-risk group was associated with lower overall survival ( $p$-value is less than 0.05) (Fig. 4C). The infiltration levels of six kinds of immune cells were extracted from the TIMER database, including B cell, CD4 T cell, CD8 T cell, Neutrophil, Macrophage and Dendritic. Analysis of the differences in the infiltration levels of the six kinds of immune cell in the highand low-risk groups showed that in the low-risk score group, all six kinds of immune cells had high levels of infiltration ( $p$-value is less than 0.05) (Fig. 4D).

\section{Aberrant expression and prognostic value analysis of hub-independent Prognostic factors in melanoma and LCK might serve as a promising indicator for remodeling TME}

The results of differential expression of three hub-independent prognostic factors in normal and tumor tissues through the TCGA cohort showed that LCK was highly expressed in tumor tissues, while KIT and EGFR were highly expressed in normal tissues (Fig. 5A). In addition, the results of Kaplan-Meier survival analysis of the three hub-independent prognostic factors, also derived from TCGA cohort, showed that high-expression of LCK was associated with prolonged overall survival, while high expression of KIT and EGFR was associated with reduced overall survival (Fig. 5B). The results of the above analysis were reproduced through the GEPIA database (Supplementary 4). Furthermore, we analyzed the correlation between the expression of the three hub-independent factors and clinical parameters, including lymph nodes, tumor topography, pathologic stage, event, gender and metastasis (Supplementary 5). The results of analysis showed that LCK expression was negatively correlated with the size of the primary tumor (tumor topography) ( $p$-value $=3.6 \mathrm{e}-05$ ) and higher degree of metastasis correlates with lower LCK expression ( $p$-value $=0.0024$ ). LCK expression was higher in surviving patients, while KIT and EGFR expression was higher in deceased patients. Through TIMER database, we analyzed the relationship of the mRNA expression of three hub-independent prognostic factors with the level of infiltration of six kinds of immune cells and tumor purity and results showed that LCK was positively correlated with the infiltration level of the six kinds of immune cells and negatively correlated with tumor purity ( $p$-value is less than 0.05) (Fig. 5C). Besides, correlation analysis of the infiltration levels of 29 immune cell calculated by the sSGSEA algorithm with the expression levels of three hub-independent prognostic factors showed that LCK is highly positively correlated with the infiltration level of 28 kinds of immune cells other than mast cells (Fig. 5D), indicating that LCK play an important role in melanoma occurrence and progression partly because of altering the level of infiltration of immune cells in TME.

\section{Correlation analysis of the LCK to common ICPs and to the proportion of TICs}

Immunotherapy such as immune checkpoint inhibitors have wide application in some solid tumors such as melanoma. However, the issue of patient responsiveness is an obstacle to their effective application. Therefore, in this study, we analyzed the relationship between the three hub-independent prognostic factors and 12 kinds of ICPS. Correlation analysis showed that LCK was significantly and positively correlated with 10 kinds of ICPs other than IL6 and CD276 (Fig. 6A). Furthermore, high expression of ICPs was observed in high LCK expression group (Fig. 6B). The results demonstrated that patients with high 
LCK expression tended to have a better immunotherapy response because of the high levels of ICPs[25]. In light of the above series of analyses, we identified LCK as a potential molecular marker for predicting immune checkpoint efficacy. After that, we divided the samples into two phenotypic cohorts according to high- and low-LCK expression and used the c2.cp.kegg.v7.4.symbols.gmt gene set for GSEA analysis, showing that the LCK high expression phenotype was mainly enriched in antigen-processing and presentation, cell adhesion molecules cams, chemokine signaling pathway, cytokine-cytokine receptor interaction, FC-GAMMA-R mediated phagocytosis, leukocyte transendothelial migration and natural killer cell mediated cytotoxicity. While LCK low expression phenotype was mainly enriched in ribosome (Fig. 6C). The results of CIBERSORT analysis showed that T cells CD8, macrophages M0, macrophages M1 and macrophages M2 were relatively high in all samples within 22 kinds of immune cell. (Supplementary $6 \mathrm{~A})$. Furthermore, analysis of the relationship between LCK expression and 22 kinds of immune components showed that the proportion of T cell CD8 was significantly higher in the LCK high-expression group, while the proportion of Macrophages M0 and Macrophages M1 were significantly lower in the LCK low-expression group (Fig. 6D). Moreover, the results of the correlation analysis between immune components and LCK expression showed that LCK expression was positively correlated with T cell regulatory (Tregs), T cell CD4 memory activation, and T cell CD8 infiltration levels. While LCK expression was negatively correlated with the infiltration level of Macrophages M0, Macrophages M2, NK cells resting (Fig. 6E). The Kaplan-Meier survival analysis based on the level of T cell CD8 and T cell CD4 memory activated immune cell infiltration showed that highe levels of infiltration of T cell CD8 and T cell CD4 memory activated were significantly associated with increased overall survival (Supplementary 6B). The above results suggested that LCK was associated with antitumor immunity in melanoma, which partially explained the association of LCK with better prognosis[27]. From the structural point of view, LCK has the typical organization found in all members of the SRC kinase family comprising an N-terminal site (SRC -homology 4, SH4 domain), a unique region, a SH3 and SH2 domain, a catalytic domain, and a short C-terminal tail[28](Supplementary 6C).

\section{Discussion}

Cancer cells must acquire phenotypic changes that allow them to evade recognition and destruction by effector cells of the immune system such as CTLs[17]. Factors that likely relate to immune escape include lack of strong cancer antigens or epitopes recognized by $T$ cells, minimal activation of cancerspecific T cells, poor infiltration of T cells into tumors, downregulation of major histocompatibility complex on cancer cells, and immunosuppressive factors and cells in the tumor microenvironment[29]. We identified a set of immune-escape related genes from the literature and overlapped them with immune-related genes to obtain core immune-escape genes. Through a series of comprehensive analysis of these immune-escape related genes, the results suggest that LCK could be a new potential target that could predict the overall survival time of melanoma patients. [25] and its high expression was significantly associated with elevated ICPs. KEGG enrichment analysis of 164 protein-coding genes, including LCK, showed that LCK is enriched in multiple immune-related pathways, including PD-L1 expression and the PD-1 checkpoint pathway in cancer, T-cell receptor signaling pathway, natural killer 
cell-mediated cytotoxicity, Th17 cell differentiation and Th1 and Th2 cell differentiation. G0 enrichment analysis showed that LCK is involved in T cell activation, $\mathrm{SH} 2$ domain binding, Protein tyrosine kinase activity, Regulation of protein kinase B signaling, Membrane region, Phosphatase binding, Protein Cterminal binding, Protein phosphorylated amino acid binding, and Phosphotyrosine residue binding. In addition, the results of the CIBERSORT analysis, which calculates the proportion of immune components in the samples, showed a higher proportion of antitumor immune cells (T cell CD8) and a lower proportion of protumor immune cells (Macrophages M0 and Macrophages M2) in the LCK high expression group. T cell CD8 is an essential component of tumor-infiltrating lymphocytes (TIL) and TME[30]. The roles and prognostic value of TILs have been extensively studied in various malignant tumors. TILs are known to have critical effects on the survival of malignant melanoma and act as prognostic markers[31-34]. TIMER website analysis showed that LCK expression was positively correlated with infiltration levels of $B$ cell $(r=0.384$, $p$-value $=3.48 \mathrm{e}-17)$, CD8 $+\mathrm{T}$ cell $(r=0.643$, $p$-value $=$ 1.87e-52), CD4 + T cell $(r=0.496, p$-value $=4.83 e-29)$, Macrophage $(r=0.357, p$-value $=5.00 \mathrm{e}-15)$, Neutrophil $(r=0.62$, p-value $=2.43 e-49)$ and Dendritic cell $(r=0.743$, p-value $=2.32 e-79)$, and negatively correlated with tumor purity $(r=-0.666, p$-value $=5.1 e-60)$. Tumor-infiltrating immune cells are closely related to tumorigenesis, angiogenesis and tumor cell growth and metastasis, which could in turn regulate the quantity and differentiation of immune cells[35]. Immune cells are the main components of $\mathrm{TME}$, and their number and status play a critical role in the progression of tumor development, invasion and metastasis[36-38]. Therefore, elucidating the infiltrating immune cells of TME may help to elucidate the potential molecular mechanisms of LCK involvement in melanoma [27]. Indeed, the fluctuating expression levels of LCK do affect the level of infiltration of immune cells in TME, which means the high level of LCK expression measured by bulk-seq is indeed a key factor that can reshape TME.

LCK (lymphocyte-specific protein tyrosine kinase) belongs to the SRC family of tyrosine kinases and has been best studied in the context of T-cell function and signaling as well as lymphocytic leukemia of the Bcell lineage[39]. LCK is mainly expressed in T cells, NK cells, brain, B cells $[39,40]$ and plays a vital role in various cellular processes such as cell cycle control, cell adhesion, motility, proliferation and differentiation[41]. This encoded protein is a key signaling molecule for selection and maturation during T cell development. LCK was strongly expressed in lymphocytes. However, tumor cells also expressed LCK, albeit to a lesser extent[42]. In recent years, it is reported that LCK has emerged as one of the key molecules regulating $T$ cell function, and studies using knockout LCK mice or LCK-deficient T cell lines surface that LCK regulates signal initiation, T cell development and T cell homeostasis and also can enhan or inhibit BCR signaling. Patients with LCK deficiency frequently present with immune dysregulation and autoimmunity. Over expression of LCK leads to large number of other diseases like cancer, asthma, diabetes 1, rheumatoid arthritis, psoriasis, systemic lupus erythematosus, inflammatory bowel diseases (crohn's disease and ulcerative colitis), organ graft rejection, atherosclerosis, hypersensitivity reactions, polyarthritis, dermatomyositis[43, 44]. Since LCK is involved in T cell proliferation and differentiation, therefore, new small molecules with LCK inhibitory activity can be of great relevance to treat $\mathrm{T}$ cell mediated diseases. It has been reported that increased LCK expression sometimes leads to colorectal cancer[45]. In addition, another report stated that inhibition or 
downregulation of LCK led to apoptosis in CLL cell lines[46]. Therefore, the application of LCK inhibitors could be an important strategy for the treatment of certain cancers[47]. However, it has also been reported that high expression of LCK is associated with increased cumulative survival in melanoma patients[48]. In another cohort survival analysis report on LCK-deficient CLL disease models in mice, results showed that LCK-KO control mice exhibited a significantly shorter median survival time compared to wild-type healthy mice over an observation period of 350 days due to T-cell deficiency and the resulting immunodeficiency[49]. Likewise, in our present study, elevated LCK expression levels in the TCGA cohort had an independent prognostic value in melanoma, and results demonstrating that upregulation of LCK expression plays a defensive role in the developmental progression of melanoma. This was reproduced in the GEPIA and the human atlas databases (HPA). LCK functions primarily in lymphocytes and is involved in transduction from the T-cell receptor complex to the nucleus, and this specific expression and function in immune cells may partially explain the phenomenon that high LCK expression is often associated with extended overall survival. On the other hand, RNA-seq is actually a kind of bulk-seq, in which various cells are mixed, so the highly expressed genes measured by RNA-seq in tumor tissues are likely to be molecules highly expressed on immune cells. In addition, since LCK plays a role in cancer cell signaling as well as in T-cell function, it will be necessary to define therapeutic strategies to selectively target LCK in tumor cells without impairing the responses of tumor infiltration lymphocytes. This is a critical issue common to other kinase inhibitors targeting signaling molecules expressed in both cancer and immune cells (e.g., B-Raf, AKT, mTOR inhibitors)[39].

\section{Conclusion}

In the present study, we conclusively identified LCK as a molecule that can remodel TME and is associated with prolonged overall survival in melanoma through a systematic analysis of immuneescape related genes, which suggest that LCK is a potential molecular marker which can predict the overall survival in melanoma. In addition, LCK targeting tumor cells will also be a promising therapeutic approach.

\section{Abbreviations}

BP, Biological process; CC, Cellular component; CIBERSOT, Cell type Identification By Estimating Relative Subsets Of RNA Transcripts; CTL, Cytotoxic T lymphocyte; GEPIA, Gene Expression Profiling Interactive Analysis;

GO, Gene Ontology; GSEA, Gene set enrichment analysis; GTEx, The Genotype-Tissue Expression; ICl, immune checkpoint inhibitor; KEGG, Kyoto Encyclopedia of Genes and Genomes; LASSO, The least absolute shrinkage and selection operator; MAF, Mutation Annotation Format; MF, Molecular function;

PPI, Protein-Protein Interaction; RPPA, Reverse Phase Protein Array; ssGSEA, Single cell gene set enrichment analysis; TCGA, The Cancer Genome Atlas; TIIC, Tumor-infiltrating immune cell; TIL, Tumorinfiltrating lymphocytes; TME, Tumor microenvironment; TRS, Training set; TES, Testing set; 


\section{Declarations}

Acknowledgements

Not applicable.

\section{Authors' contributions}

DLZ and ZZ clutched the article and designed the research idea. FKD, YC, XY, JS, ZGX, ZMY and SXX collected and organized the study data, and preprocessed the data in statistical software. MTX, YSZ, YZ, QLW, JL and YZ visualized the results and embellished and typeset the images in the software. SD, XW and MXL jointly revised the article. All authors read and approved the final manuscript.

\section{Funding}

This work was supported by National Natural Science Foundation of China (No. 81972643), Sichuan Science and Technology Project (2021YJ0201) and Luxian People's Government and Southwest Medical University Scientific and Technological Achievements Transfer and Transformation Strategic Cooperation Project (2019LXXNYKD-07).

\section{Availability of data and materials}

The datasets used and/or analyzed during the current study are available from the corresponding author on reasonable request.

\section{Ethics approval and consent to participate}

Not applicable

\section{Consent for publication}

Not applicable

\section{Competing interests}

The authors declare that they do not have any conflicts of interest.

\section{References}

1. Perez $\mathrm{E}$, Reyes $\mathrm{O}$, Ventura S: Convolutional neural networks for the automatic diagnosis of melanoma: An extensive experimental study. Med Image Anal 2021, 67:101858.

2. 2.. In: Cutaneous Melanoma: Etiology and Therapy. Edited by Ward WH, Farma JM. Brisbane (AU); 2017.

3. Siegel RL, Miller KD, Fuchs HE, Jemal A: Cancer Statistics, 2021. CA Cancer J Clin 2021, 71(1):7-33. 
4. Pogorzelska-Antkowiak A, Calik J: Mimics of melanoma in reflectance confocal microscopy. Int J Dermato/2021, 60(5):540-546.

5. Krattinger R, Ramelyte E, Dornbierer J, Dummer R: Is single versus combination therapy problematic in the treatment of cutaneous melanoma? Expert Rev Clin Pharmacol 2021, 14(1):9-23.

6. Hou J, Karin M, Sun B: Targeting cancer-promoting inflammation - have anti-inflammatory therapies come of age? Nat Rev Clin Oncol 2021, 18(5):261-279.

7. O'Donnell JS, Teng MWL, Smyth MJ: Cancer immunoediting and resistance to T cell-based immunotherapy. Nat Rev Clin Oncol 2019, 16(3):151-167.

8. Zuo S, Wei M, Wang S, Dong J, Wei J: Pan-Cancer Analysis of Immune Cell Infiltration Identifies a Prognostic Immune-Cell Characteristic Score (ICCS) in Lung Adenocarcinoma. Front Immuno/ 2020, 11:1218.

9. Vanichapol T, Chutipongtanate S, Anurathapan U, Hongeng S: Immune Escape Mechanisms and Future Prospects for Immunotherapy in Neuroblastoma. Biomed Res Int 2018, 2018:1812535.

10. Hanahan D, Coussens LM: Accessories to the crime: functions of cells recruited to the tumor microenvironment. Cancer Cell 2012, 21(3):309-322.

11. Bi KW, Wei XG, Qin XX, Li B: BTK Has Potential to Be a Prognostic Factor for Lung Adenocarcinoma and an Indicator for Tumor Microenvironment Remodeling: A Study Based on TCGA Data Mining. Front Oncol 2020, 10:424.

12. Quail DF, Joyce JA: Microenvironmental regulation of tumor progression and metastasis. Nat Med 2013, 19(11):1423-1437.

13. Wood SL, Pernemalm M, Crosbie PA, Whetton AD: The role of the tumor-microenvironment in lung cancer-metastasis and its relationship to potential therapeutic targets. Cancer Treat Rev 2014, 40(4):558-566.

14. Liu B, Wang Z, Gu M, Zhao C, Ma T, Wang J: GEO Data Mining Identifies OLR1 as a Potential Biomarker in NSCLC Immunotherapy. Front Oncol 2021, 11:629333.

15. Newman AM, Liu CL, Green MR, Gentles AJ, Feng W, Xu Y, Hoang CD, Diehn M, Alizadeh AA: Robust enumeration of cell subsets from tissue expression profiles. Nat Methods 2015, 12(5):453-457.

16. Barbie DA, Tamayo P, Boehm JS, Kim SY, Moody SE, Dunn IF, Schinzel AC, Sandy P, Meylan E, Scholl $C$ et al: Systematic RNA interference reveals that oncogenic KRAS-driven cancers require TBK1. Nature 2009, 462(7269):108-112.

17. Lawson KA, Sousa CM, Zhang X, Kim E, Akthar R, Caumanns JJ, Yao Y, Mikolajewicz N, Ross C, Brown KR et al: Functional genomic landscape of cancer-intrinsic evasion of killing by $\mathrm{T}$ cells. Nature 2020, 586(7827):120-126.

18. Mayakonda A, Lin DC, Assenov Y, Plass C, Koeffler HP: Maftools: efficient and comprehensive analysis of somatic variants in cancer. Genome Res 2018, 28(11):1747-1756.

19. Gao J, Aksoy BA, Dogrusoz U, Dresdner G, Gross B, Sumer SO, Sun Y, Jacobsen A, Sinha R, Larsson E et al: Integrative analysis of complex cancer genomics and clinical profiles using the cBioPortal. Sci 
Signal 2013, 6(269):pl1.

20. Zhao Y, Zhao Q, Kaboli PJ, Shen J, Li M, Wu X, Yin J, Zhang H, Wu Y, Lin L et al: m1A Regulated Genes Modulate PI3K/AKT/mTOR and ErbB Pathways in Gastrointestinal Cancer. Trans/ Onco/2019, 12(10):1323-1333.

21. Lei Y, Yu T, Li C, Li J, Liang Y, Wang X, Chen Y, Wang X: Expression of CAMK1 and its association with clinicopathologic characteristics in pancreatic cancer. J Cell Mol Med 2021, 25(2):1198-1206.

22. Xiao B, Liu L, Li A, Xiang C, Wang P, Li H, Xiao T: Identification and Verification of Immune-Related Gene Prognostic Signature Based on ssGSEA for Osteosarcoma. Front Oncol 2020, 10:607622.

23. Li T, Fan J, Wang B, Traugh N, Chen Q, Liu JS, Li B, Liu XS: TIMER: A Web Server for Comprehensive Analysis of Tumor-Infiltrating Immune Cells. Cancer Res 2017, 77(21):e108-e110.

24. Tang Z, Li C, Kang B, Gao G, Li C, Zhang Z: GEPIA: a web server for cancer and normal gene expression profiling and interactive analyses. Nucleic Acids Res 2017, 45(W1):W98-W102.

25. Xu F, Shen J, Xu S: Integrated Bioinformatical Analysis Identifies GIMAP4 as an Immune-Related Prognostic Biomarker Associated With Remodeling in Cervical Cancer Tumor Microenvironment. Front Cell Dev Biol 2021, 9:637400.

26. Kang K, Xie F, Mao J, Bai Y, Wang X: Significance of Tumor Mutation Burden in Immune Infiltration and Prognosis in Cutaneous Melanoma. Front Oncol 2020, 10:573141.

27. Cao Y, Jiao N, Sun T, Ma Y, Zhang X, Chen H, Hong J, Zhang Y: CXCL11 Correlates With Antitumor Immunity and an Improved Prognosis in Colon Cancer. Front Cell Dev Biol 2021, 9:646252.

28. Ventimiglia LN, Alonso MA: The role of membrane rafts in Lck transport, regulation and signalling in T-cells. Biochem J 2013, 454(2):169-179.

29. Kim JM, Chen DS: Immune escape to PD-L1/PD-1 blockade: seven steps to success (or failure). Ann Oncol 2016, 27(8):1492-1504.

30. Oshi M, Asaoka M, Tokumaru Y, Yan L, Matsuyama R, Ishikawa T, Endo I, Takabe K: CD8 T Cell Score as a Prognostic Biomarker for Triple Negative Breast Cancer. Int J Mol Sci 2020, 21(18).

31. Lee KH, Kim EY, Yun JS, Park YL, Do SI, Chae SW, Park CH: The prognostic and predictive value of tumor-infiltrating lymphocytes and hematologic parameters in patients with breast cancer. BMC Cancer 2018, 18(1):938.

32. Lee N, Zakka LR, Mihm MC, Jr., Schatton T: Tumour-infiltrating lymphocytes in melanoma prognosis and cancer immunotherapy. Pathology 2016, 48(2):177-187.

33. Mahmoud SM, Paish EC, Powe DG, Macmillan RD, Grainge MJ, Lee AH, Ellis IO, Green AR: Tumorinfiltrating CD8 + lymphocytes predict clinical outcome in breast cancer. J Clin Oncol 2011, 29(15):1949-1955.

34. Schatton T, Scolyer RA, Thompson JF, Mihm MC, Jr.: Tumor-infiltrating lymphocytes and their significance in melanoma prognosis. Methods Mol Biol 2014, 1102:287-324.

35. Di Caro G, Marchesi F, Laghi L, Grizzi F: Immune cells: plastic players along colorectal cancer progression. J Cell Mol Med 2013, 17(9):1088-1095. 
36. Zheng C, Zheng L, Yoo JK, Guo H, Zhang Y, Guo X, Kang B, Hu R, Huang JY, Zhang Q et al: Landscape of Infiltrating T Cells in Liver Cancer Revealed by Single-Cell Sequencing. Cell2017, 169(7):13421356 e1316.

37. Garnelo M, Tan A, Her Z, Yeong J, Lim CJ, Chen J, Lim KH, Weber A, Chow P, Chung A et al: Interaction between tumour-infiltrating $B$ cells and $T$ cells controls the progression of hepatocellular carcinoma. Gut 2017, 66(2):342-351.

38. Ouyang FZ, Wu RQ, Wei Y, Liu RX, Yang D, Xiao X, Zheng L, Li B, Lao XM, Kuang DM: Dendritic cellelicited B-cell activation fosters immune privilege via IL-10 signals in hepatocellular carcinoma. Nat Commun 2016, 7:13453.

39. Bommhardt U, Schraven B, Simeoni L: Beyond TCR Signaling: Emerging Functions of Lck in Cancer and Immunotherapy. Int J Mol Sci 2019, 20(14).

40. Kumar Singh P, Kashyap A, Silakari O: Exploration of the therapeutic aspects of Lck: A kinase target in inflammatory mediated pathological conditions. Biomed Pharmacother 2018, 108:1565-1571.

41. Gorassini A, Verardo G, Bortolomeazzi R: Polymeric reversed phase and small particle size silica gel solid phase extractions for rapid analysis of sterols and triterpene dialcohols in olive oils by GC-FID. Food Chem 2019, 283:177-182.

42. Weisse J, Rosemann J, Muller L, Kappler M, Eckert AW, Glass M, Misiak D, Huttelmaier S, Ballhausen WG, Hatzfeld M et al: Identification of lymphocyte cell-specific protein-tyrosine kinase (LCK) as a driver for invasion and migration of oral cancer by tumor heterogeneity exploitation. Mol Cancer 2021, 20(1):88.

43. Sabat M, Vanrens JC, Brugel TA, Maier J, Laufersweiler MJ, Golebiowski A, De B, Easwaran V, Hsieh LC, Rosegen J et al: The development of novel 1,2-dihydro-pyrimido[4,5-c]pyridazine based inhibitors of lymphocyte specific kinase (Lck). Bioorg Med Chem Lett 2006, 16(16):4257-4261.

44. Takayama T, Umemiya H, Amada H, Yabuuchi T, Koami T, Shiozawa F, Oka Y, Takaoka A, Yamaguchi A, Endo $M$ et al: Ring-fused pyrazole derivatives as potent inhibitors of lymphocyte-specific kinase (Lck): Structure, synthesis, and SAR. Bioorg Med Chem Lett 2010, 20(1):112-116.

45. Chakraborty G, Rangaswami H, Jain S, Kundu GC: Hypoxia regulates cross-talk between Syk and Lck leading to breast cancer progression and angiogenesis. J Biol Chem 2006, 281(16):11322-11331.

46. Talab F, Allen JC, Thompson V, Lin K, Slupsky JR: LCK is an important mediator of B-cell receptor signaling in chronic lymphocytic leukemia cells. Mol Cancer Res 2013, 11(5):541-554.

47. Harr MW, Caimi PF, McColl KS, Zhong F, Patel SN, Barr PM, Distelhorst CW: Inhibition of Lck enhances glucocorticoid sensitivity and apoptosis in lymphoid cell lines and in chronic lymphocytic leukemia. Cell Death Differ 2010, 17(9):1381-1391.

48. Cancer Genome Atlas N: Genomic Classification of Cutaneous Melanoma. Cell 2015, 161(7):16811696.

49. Marklin M, Fuchs AR, Tandler C, Heitmann JS, Salih HR, Kauer J, Quintanilla-Martinez L, Wirths S, Kopp HG, Muller MR: Genetic Loss of LCK Kinase Leads to Acceleration of Chronic Lymphocytic Leukemia. Front Immunol 2020, 11:1995. 
Figures

A Immune-related gene

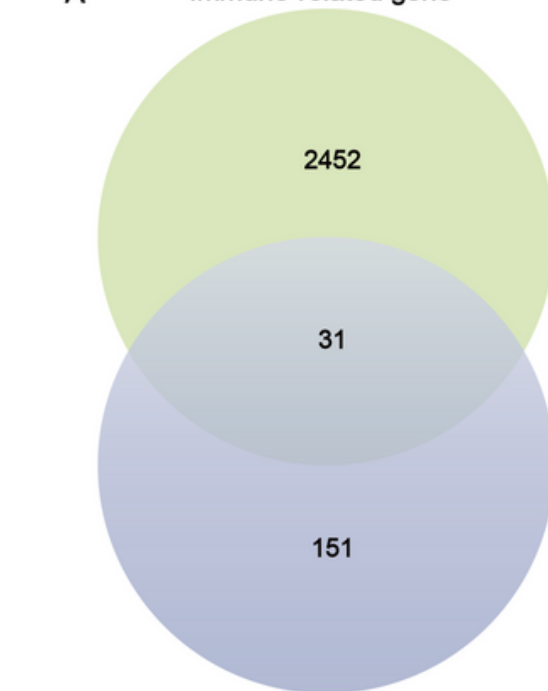

Immune-escape related gene

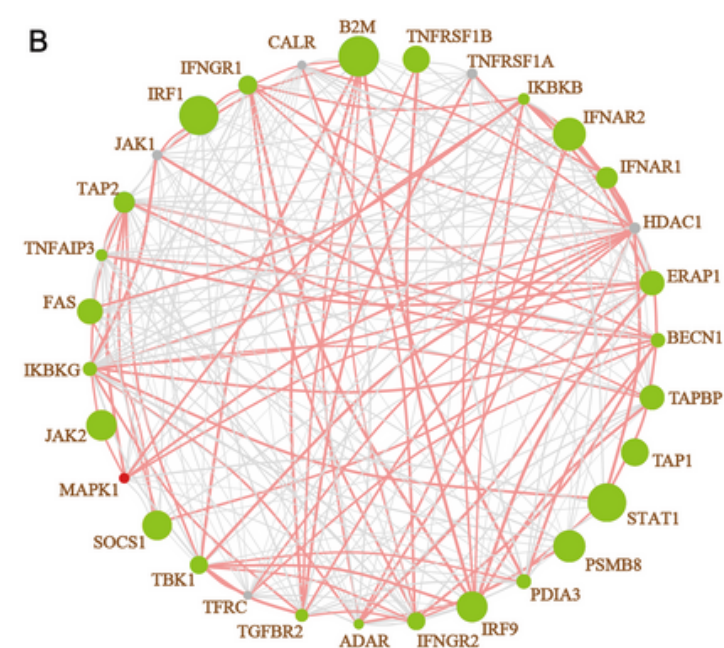

No significant differences in Kaplan-Meier survival curves

Risk factor for OS

Favorable factor for OS

logrank test, $P$ value

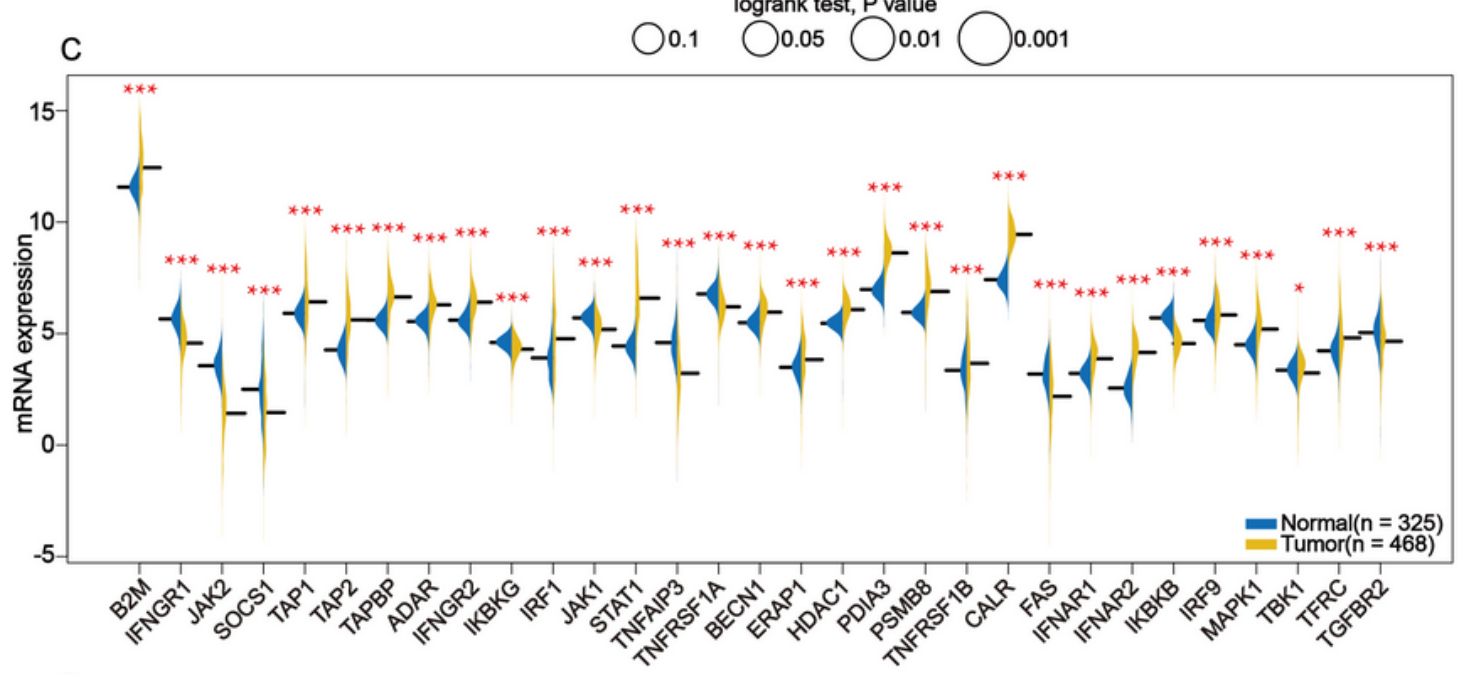

D

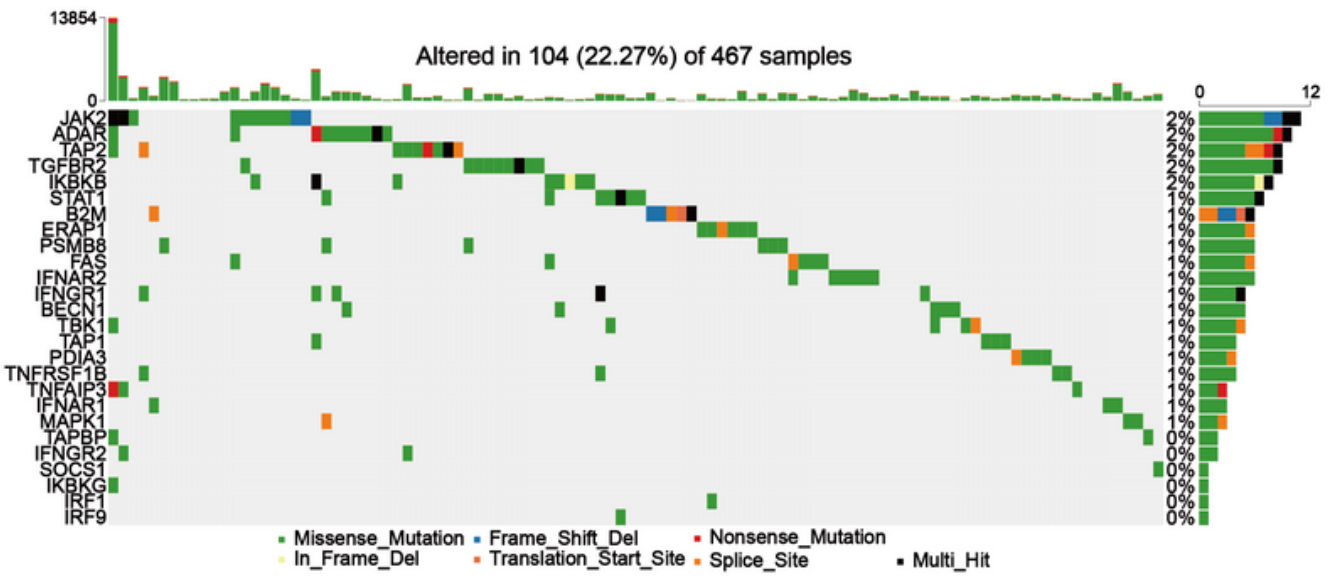

Figure 1

Most crossover genes have significant prognostic value in melanoma and differentially expressed in normal and tumor tissues. (A) 31 core immune-escape related genes were identified as shown in Venn diagram. (B) Prognostic analysis of 31 core immune-escape related genes. The results showed that all 26 
genes were favorable prognostic factors, except for CALR, TNFRSF1A, TFRC, and HDAC1 prognostic analysis results did not differ significantly, and MAPK1, which was an unfavorable prognostic factor. Green dots represent favorable prognostic factors, red dots represent unfavorable prognostic factors, and grey dots represent genes that are not significantly different in the Kaplan-Meier survival curve. The bigger the dot is, the smaller the $\mathrm{P}$ value is. The line between the different dots represents the correlation between them. The red line indicates a positive correlation between them; The blue line indicates a negative correlation between them; The grey line indicates that the two are not related. $P$ value calculated by log-rank test and the correlation coefficient between the core immune-escape related genes were evaluated using Spearman's correlation analysis. (C) Normalized expression across tissue and cancer types for 31 core immune-escape related genes. The normalized expression of RNA sequence (TPM) detected from the tumor (TCGA) and normal (GTEx) database, which contain 468 complete tumor patients and 325 normal samples. All intersecting genes were differentially expressed in normal and tumor tissues, with significantly different results. Blue and yellow half-violins represented normal and tumor samples, respectively. ${ }^{\star} \mathrm{P}<.05,{ }^{\star} \mathrm{P}<.01,{ }^{\star \star \star} \mathrm{P}<.001$, by two-tailed $\mathrm{t}$ test. (D) Waterfall plot of detailed mutation information of 26 genes after initial screening in each sample, with various color annotations to distinguish different mutation types. In summary, they are less likely to have mutations. 
A EGFR tyrosine kinase inhibitor resistance KEGG enrichment analysis PI3K-Akt signaling pathway Cellular senescence FoxO signaling pathwayHIF-1 signaling pathway Apoptosis Autophagy - anima AMPK signaling pathway Choline metabolism in cancer PD-L1 expression and path-1 checkpoint. P53 signaling pathway Fc epsilon RI signaling pathway Chemokine signaling pathway. $T$ cell receptor signaling pathway. Natural killer cell mediated cytotoxicity. Th17 cell differentiation Th1 and Th2 cell differentiation NF-kappa B signaling pathway.

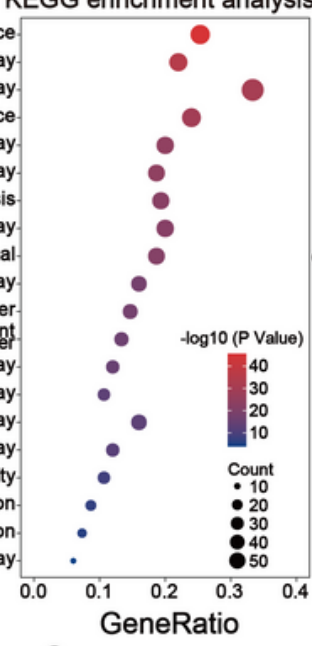

C

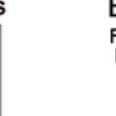

B

GO functional enrichment analysis

Regulation of apoptotic signaling pathway

Regulation of mitochondrion organization

Regulation of membrane permeability

Regulation of intracellular transport

Regulation of mitochondrial membrane permeability

Cell aging
Regulation of protein kinase $B$ signaling

T cell activation

Chromosome, telomeric regio

Cyclin-dependent protein kinase holoenzyme complex

Transferase complex

nelle outer membran

Outer membrane

Chromosomal region

Focal adhesion

Phosphatase bindin

Ubiquitin-like protein ligase binding

Phosphoprotein bindin

Protein C-terminus binding

Protein phosphorylated amino acid binding

DNA

Phosphotyrosine residue binding

艿

है।

SH2 domain binding

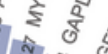

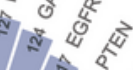

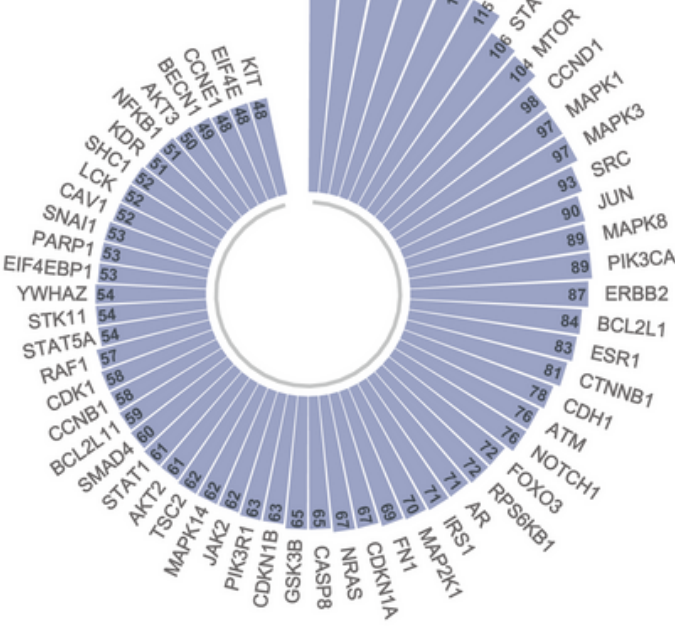

D

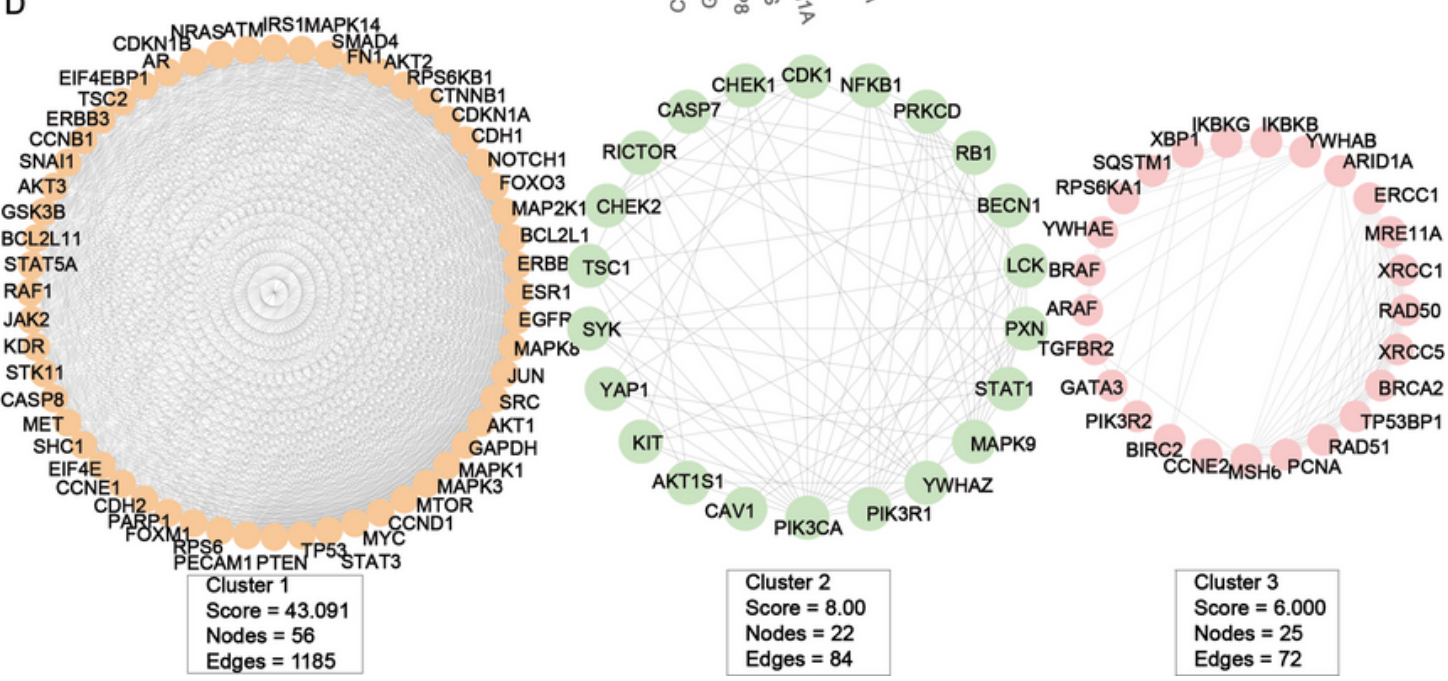

Figure 2

Pathway and functional enrichment analysis of protein-coding genes. (A) The screened differential proteins were utilized for GO and KEGG enrichment analysis through R package "ClusterProfiler". These differential proteins are enriched in multiple immune-related pathways and functional modules. (B) The top 60 nodes ordered by the connectivity of nodes in Protein-Protein Interaction. These top 60 nodes will 
be included in the further analysis. (C) Three important subnetworks in PPI networks which is tightly connected areas.

\begin{tabular}{|c|c|c|c|c|}
\hline Gene & HR & $95 \% \mathrm{Cl}$ & Hazard ratio & $\mathrm{P}$ value \\
\hline FOXM1 & 1.449 & $(1.177-1.784)$ & $1 \longmapsto \longrightarrow$ & $0.00048^{* * * *}$ \\
\hline KIT & 1.114 & (1.040-1.192) & 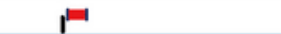 & 0.002 ** \\
\hline BRD4 & 1.827 & $(1.242-2.686)$ & $1 \longmapsto$ & 0.00219 ** \\
\hline EGFR & 1.135 & $(1.046-1.231)$ & $1=$ & 0.00227 ** \\
\hline RAB25 & 1.078 & (1.023-1.135) & 惩 & 0.00474 ** \\
\hline CCNE1 & 1.337 & $(1.087-1.644)$ & 1 & 0.00587 ** \\
\hline LCK & 0.900 & $(0.832-0.973)$ & 昌 & 0.00825 ** \\
\hline EIF4G1 & 1.521 & (1.096-2.112) & $1 \longmapsto$ & 0.01223 * \\
\hline ACACA & 1.401 & $(1.063-1.845)$ & $I_{\longmapsto}=$ & 0.01659 * \\
\hline SMAD1 & 1.230 & (1.037-1.458) & $1-1=-1$ & 0.01715 * \\
\hline IRS1 & 1.156 & (1.015-1.317) & $t=-1$ & 0.02852 * \\
\hline EEF2 & 1.324 & (1.027-1.706) & $\longmapsto$ & 0.03041 * \\
\hline KDR & 1.196 & $(1.010-1.415)$ & $1=-1$ & 0.03816 * \\
\hline CDKN1B & 0.760 & $(0.580-0.996)$ & $\mapsto-1$ & 0.04656 * \\
\hline RICTOR & 0.841 & $(0.708-1.000)$ & $\mathrm{F}=\mathrm{-1}$ & $0.04996 *$ \\
\hline
\end{tabular}

B
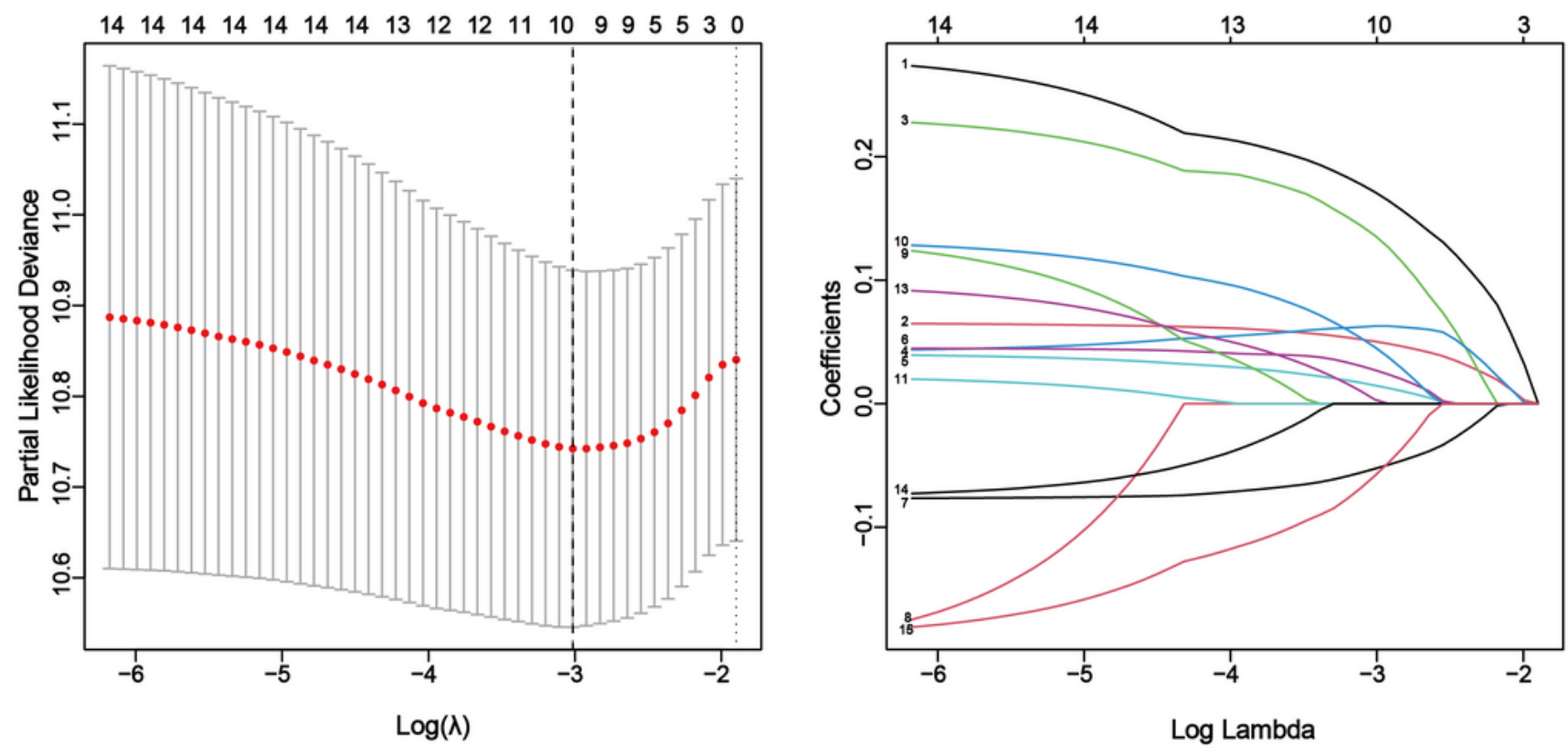

C

D

\begin{tabular}{|c|c|c|c|c|}
\hline Gene & HR & $95 \% \mathrm{Cl}$ & Hazard ratio & $P$ value \\
\hline FOXM1 & 1.381 & $(1.121-1.701)$ & $1 \longmapsto \longrightarrow$ & $0.002 * *$ \\
\hline KIT & 1.089 & $(1.015-1.168)$ & PEA & $0.018 *$ \\
\hline EGFR & 1.108 & $(1.012-1.213)$ & 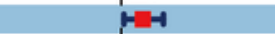 & $0.027 *$ \\
\hline LCK & 0.912 & $(0.833-0.999)$ & 낼 & $0.047 *$ \\
\hline SMAD1 & 1.180 & $(0.967-1.441)$ & 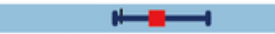 & 0.103 \\
\hline \multirow[t]{2}{*}{ RICTOR } & 0.865 & $(0.727-1.030)$ & 븜ㄱ & 0.104 \\
\hline & & & 1.0 & \\
\hline
\end{tabular}

57

3

MultiCox result

PPI

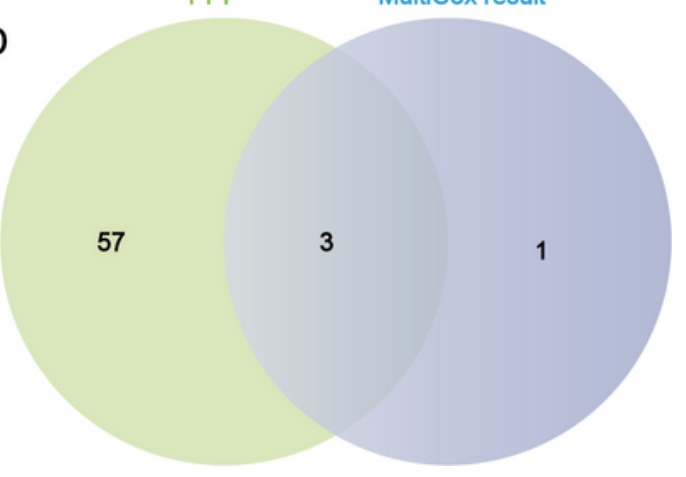

Figure 3

Identification of independent prognostic factors with prognostic value including LCK, KIT and EGFR. (A) Univariate cox regression analysis for the survival of melanoma patients was performed to determine the significant factors among 164 differentially expressed proteins, listing the results of univariate cox 
regression, which contains 15 protein-coding genes in all. (B) The least absolute shrinkage and selection operator (LASSO) regression analysis for the prognostic factors obtained from the results of the univariate cox regression analysis, LASSO coefficient profiles of the 16 prognostic factors in TCGA-SKCM and coefficient profile plot was generated against the log (lambda) sequence. At last, 10 signature protein-coding genes were included in further analysis. (C) The seven protein-coding genes of multivariate cox proportional-hazards model. There were significant differences in results for four of the genes, which means they are independent prognostic factors with prognostic value. (D) Hub-independent prognostic factors were selected by overlaying key proteins in the PPI and independent prognostic factors obtained by multivariate cox regression. Three protein-coding genes were obtained and are regarded as hubindependent prognostic factors. 


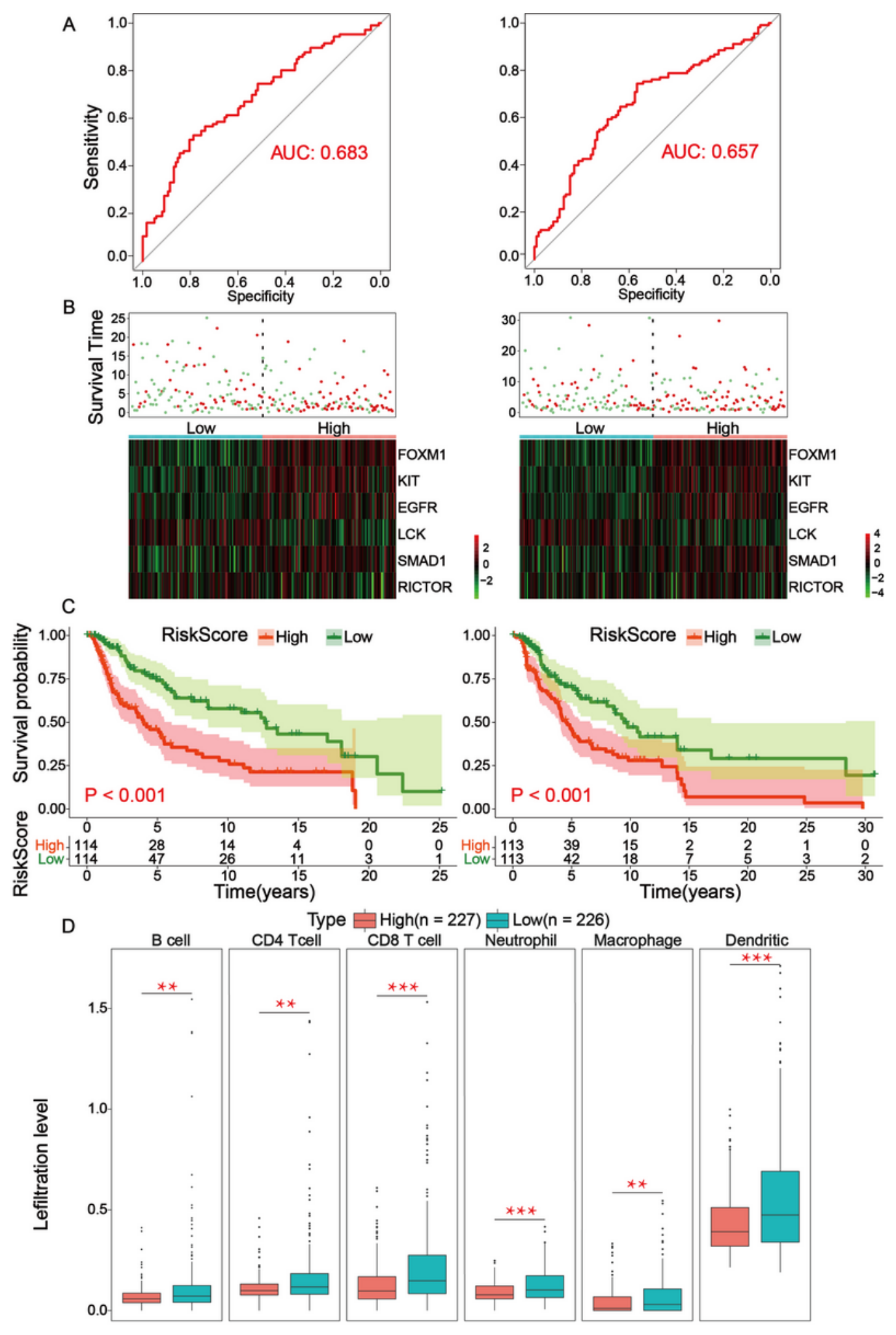

\section{Figure 4}

Low-risk group in prognostic risk model associated with longer overall survival. (A) In the TRS and TES, the ROC curve is used to describe the accuracy of the model, with a higher AUC value representing a more accurate model. the AUC value is quantified by the risk score calculated from the COEF value in the results of the multivariate cox proportional-hazards model. The AUC values of the ROC curves for the training and test groups are 0.683 and 0.657 , respectively, indicating that the risk model is relatively 
accurate. (B) Risk factor analysis in TRS and TES, the samples are divided into two groups of high- and low-risk according to the risk score, with green dots representing surviving samples and red dots representing dead samples. As the risk score increases, the number of dead samples also increases. The heatmap shows the expression of the six factors in the multivariate results in the high- and low-risk groups. Green area represents low expression; red area represents high expression. As the risk score increased, the mortality sample also increased, indicating that the risk score model does predict patient prognosis. (C) Survival curves based on high- and low-risk score for the training and test sets. In both the training and test sets, low-risk scores were associated with elevated overall survival. (D) Differential expression analysis of six immune cells in high- and low-risk groups, red boxes represent the high-risk group, blue boxes represent the low-risk group. Low-risk subgroups have higher infiltration levels of immune cells infiltrating into the TME. 

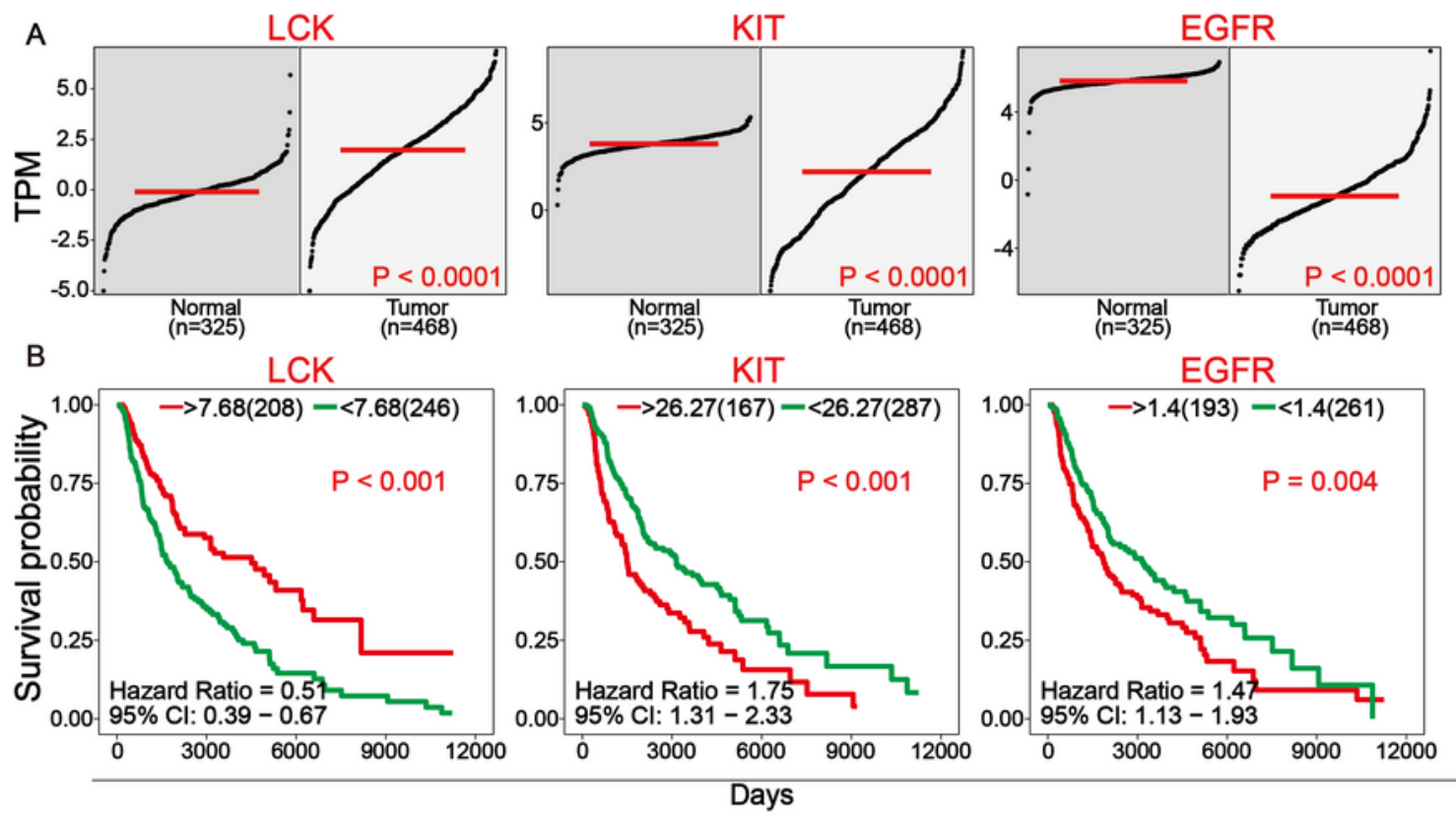

C

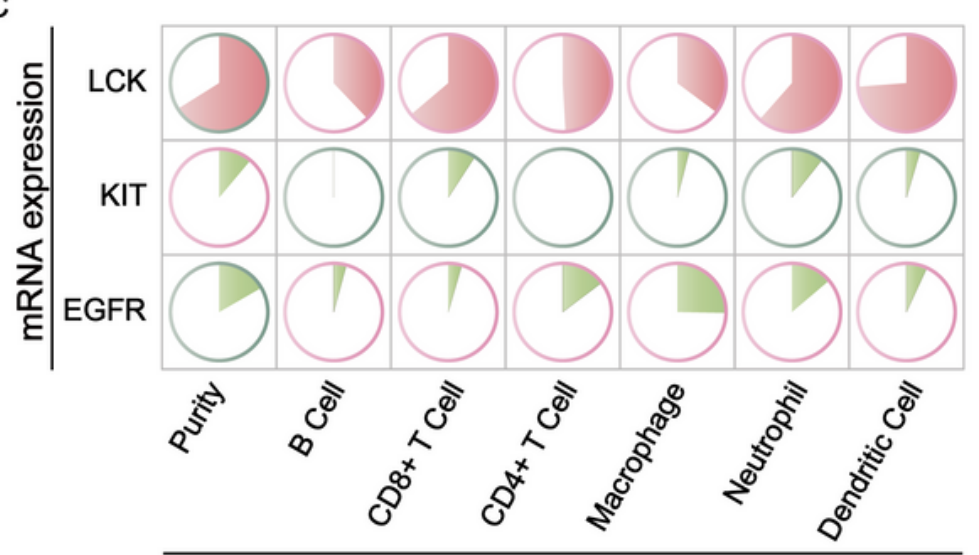

$$
|R|>0.3
$$

$|\mathrm{R}|<0.3$

\section{Positive correlation}

Negative correlation

D

Infiltration Level

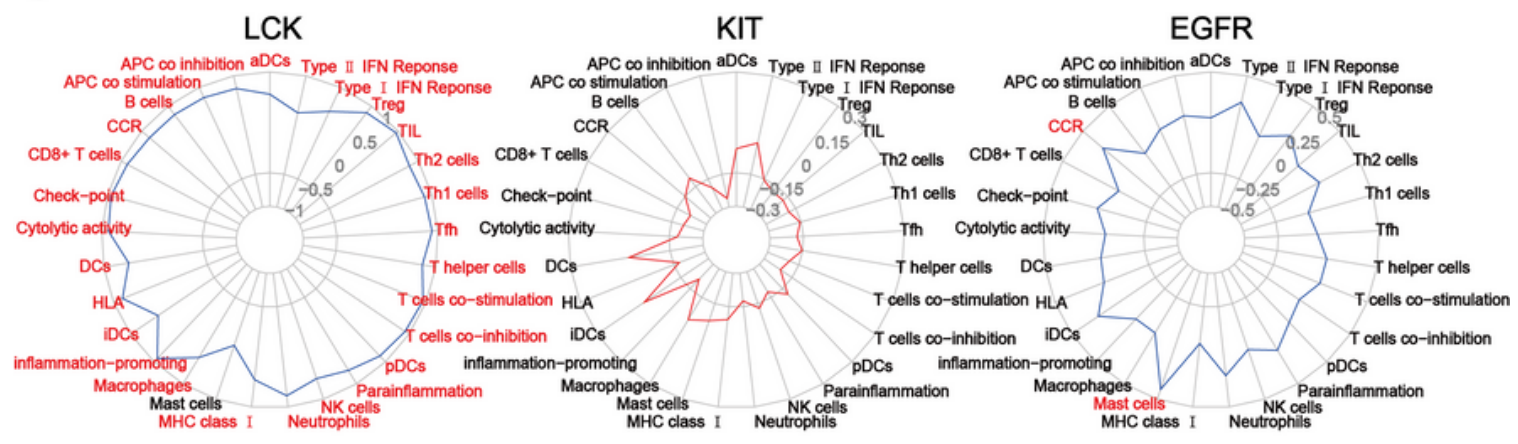

Immune cell : $\mathrm{P}$ value $<0.05$ and $|\mathrm{R}|>0.3$

Immune cell : $P$ value $<0.05$ or $|R|>0.3$

\section{Figure 5}

LCK is a hub-independent prognostic factor with potential to reshape TME. (A) Differential expression analysis of three hub-independent prognostic factors in tumor and normal samples. LCK is highly expressed in tumors while KIT and EGFR are highly expressed in normal tissues. (B) Kaplan-Meier Survival analysis of three hub- independent prognostic factors based on high and low expression. High expression of LCK was associated with prolonged overall survival, whereas high expression of KIT and 
EGFR was associated with shortened overall survival. (C) Correlation analysis of the expression levels of three hub-independent prognostic factors with the infiltration levels of six kinds of immune cells and tumor purity. The results showed that the expression level of LCK was highly positively correlated with the infiltration level of six kinds of immune cells and highly negatively correlated with tumor purity, revealing the key role of LCK in TME. The pink border represents the absolute value of $r$ correlation coefficient is greater than 0.3 , and the green border represents the absolute value of $r$ correlation coefficient is less than 0.3; the pink border represents the mRNA levels of three hub-independent prognostic factors are positively correlated with the level of six kinds of immune cell infiltration and tumor purity, and the green border represents the mRNA levels of three hub-independent prognostic factors are positively correlated with the level of six kinds of immune cell infiltration and tumor purity. negative correlation. (D) Correlation analysis of the expression levels of three hub-independent prognostic factors with the infiltration levels of 29 kinds of immune cells. Except for mast cells, LCK expression was highly positively correlated with the level of infiltration of the remaining 28 kinds of immune cell types. 
A

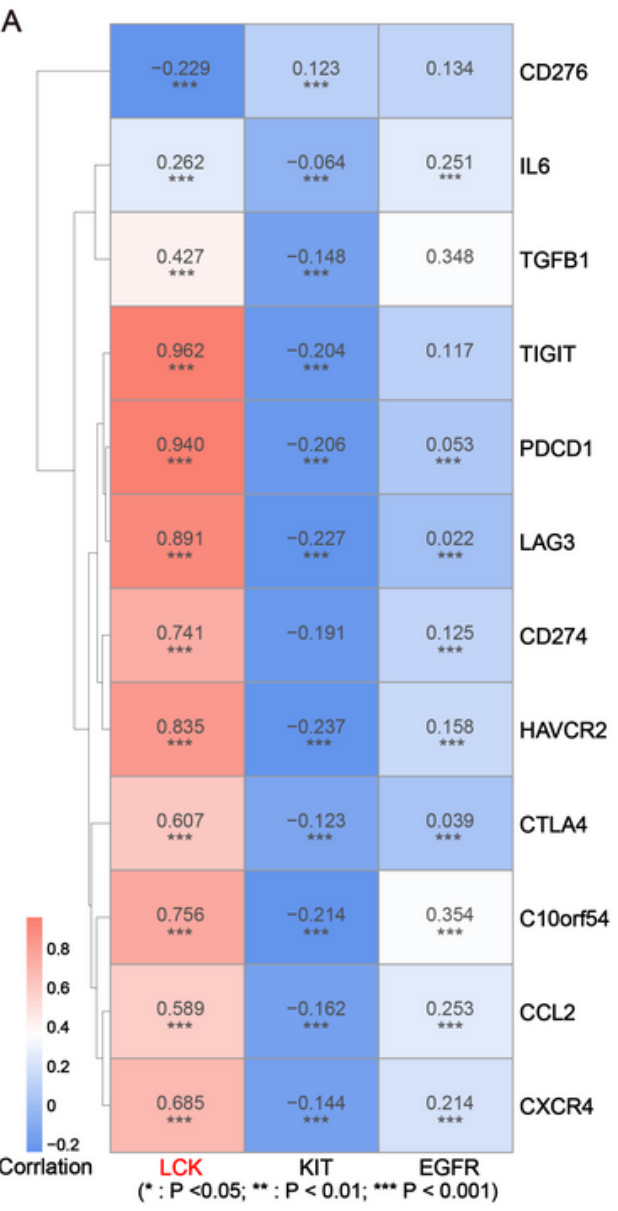

B

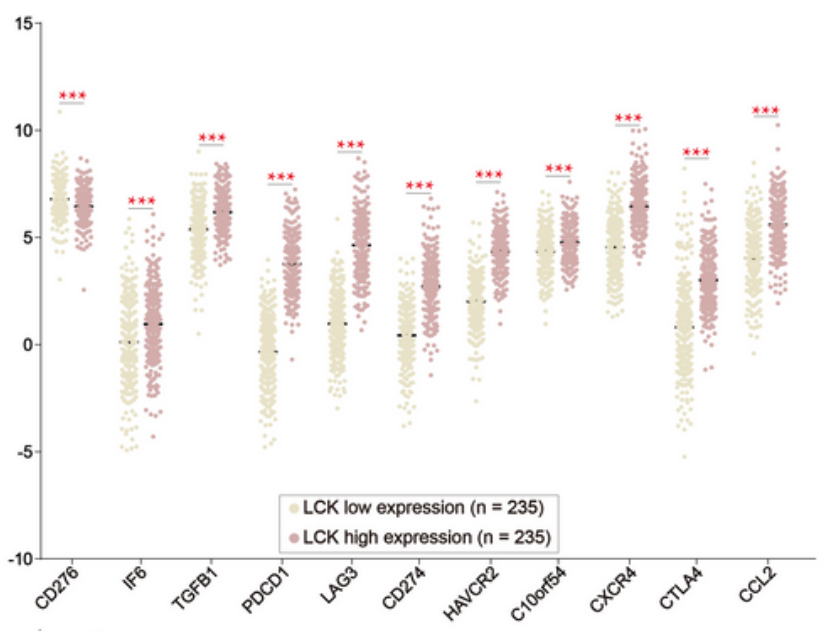

C

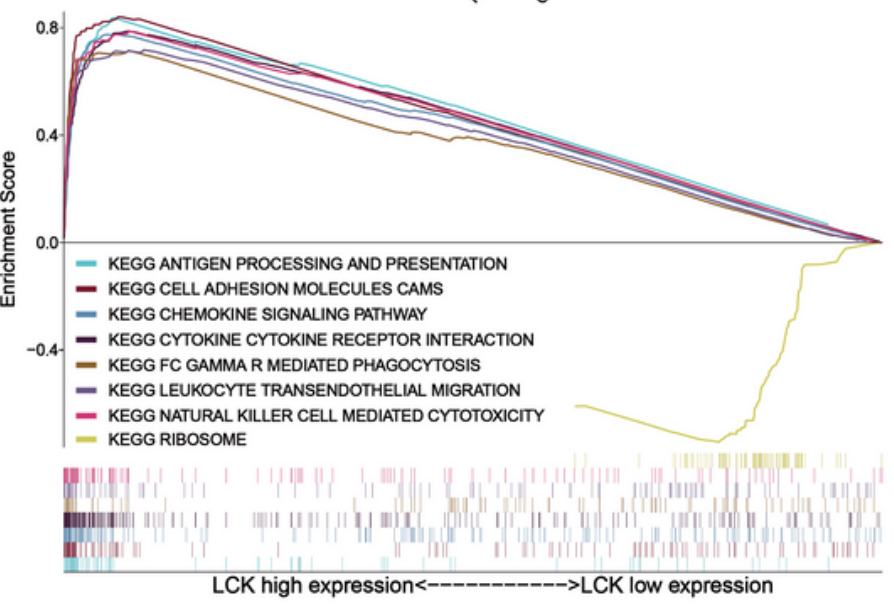

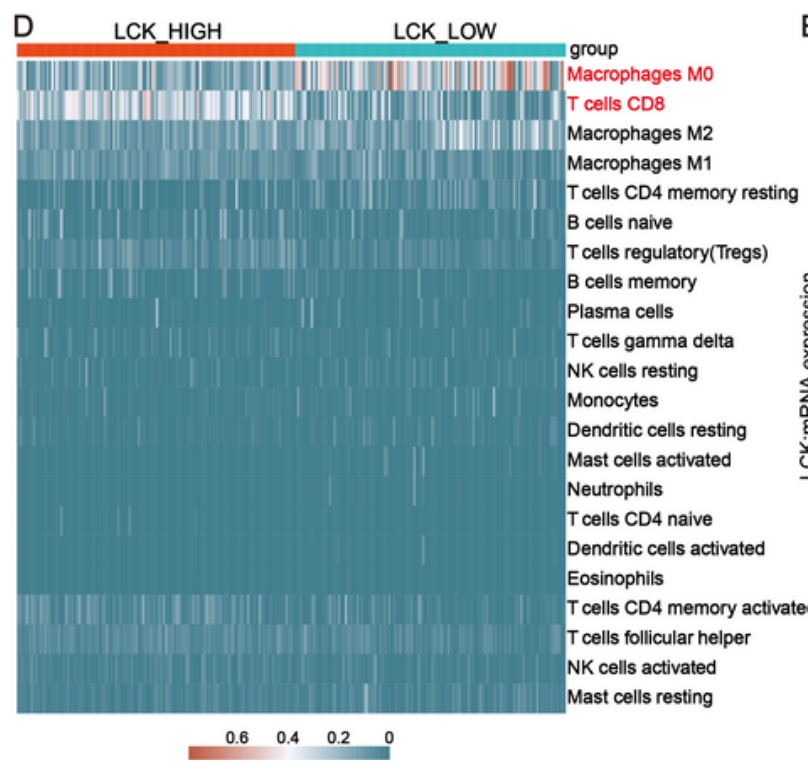

E

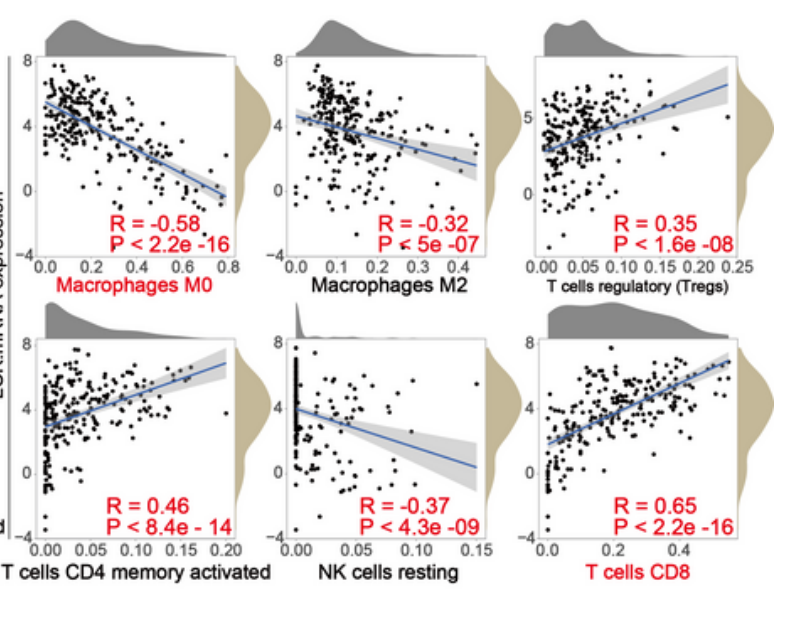

$\begin{array}{llllllllll}0.4 & 0.2 & 0 & 0\end{array}$

\section{Figure 6}

TIC profile in melanoma samples and correlation analysis, and correlation of TICs proportion and common ICPs with LCK expression. (A) Correlation of LCK with the common ICPs. The heatmap shows the results of the correlation analysis. Red area represents a positive correlation; blue area represents a negative correlation. With the exception of CD276 and IL6, LCK expression was highly positively correlated with the remaining 10 common ICPs. (B) Analysis of differential expression of ICPs in LCK 
high- and low-expression cohorts. Light gray dots represent LCK low-expression cohort; brown dots represent LCK high-expression cohort. The analysis showed that 11 kinds of common ICPs, except CD276, were highly expressed in the LCK high-expression group. (C) Gene set enrichment analysis based on high and low LCK expression typing. The LCK high-expression group genes had principal enrichment in multiple immune-related pathway. (D) The difference of proportion of immune cell between LCK high expression sample and LCK low expression sample. Brown represents a high percentage of immune cells; marine blue represents a low percentage of immune cells. T cell CD8 immune cell fraction was higher in the LCK high expression group, while Macrophages M0 and Macrophages M2 immune cell fraction was higher in the LCK low expression group. (E) Correlation analysis of the percentage of 22 kinds of immune cells with LCK expression. LCK expression was significantly and positively correlated with $\mathrm{T}$ cell regulatory, T cell CD4 memory activated and T cell CD8 infiltration levels, while it was negatively correlated with Macrophages M0, Macrophages M2 and NK cell resting.

\section{Supplementary Files}

This is a list of supplementary files associated with this preprint. Click to download.

- Figurelegendsofsupplementaryfigures.docx

- SUPPLEMENTARY1.png

- SUPPLEMENTARY2.png

- SUPPLEMENTARY3.png

- SUPPLEMENTARY4.png

- SUPPLEMENTARY5.png

- SUPPLEMENTARY6.png 